recorded in $72 \%$, and a viral illness or fever preceded the headaches in $50 \%$. Apart from 2 patients showing unidentified bright objects on MRI, neuroimaging studies were negative. The headaches had characteristics of migraine with aura, unilateral location, pulsating quality, nausea, and photophobia, but unlike typical migraine, the headache syndrome was not chronic. The syndrome resolved within 3 months. The cause is undetermined. (Berg MJ, Williams LS. The transient syndrome of headache with neurologic deficits and CSF lymphocytosis. Neurology September 1995;45:1648-1654). (Reprints: Dr Michel J Berg, Box 673, Department of Neurology, University of Rochester Medical Center, 60 Elmwood Avenue, Rochester, NY 14642).

COMMENT. The differential diagnoses listed by the authors for this syndrome include Lyme neuroborreliosis, neurosyphilis, neurobrucellosis, mycoplasma infection, granulomatous meningitis, neoplastic meningitis, autoimmune disease, HIV meningitis, and a side effect of cerebral angiography. Other illnesses with similar features are hemiplegic migraine, seizure disorder, and recurrent aseptic

meningitis (Mollaret's meningitis). The syndrome is self limited, and a viral etiology seems likely.

\title{
NEONATAL ENCEPHALOPATHY
}

\section{ANTEPARTUM CAUSES OF ENCEPHALOPATHY}

Adverse factors in the family and maternal history, pregnancy, and birth related to the occurrence of neonatal encephalopathy (NE) in full term newborn infants were evaluated in a matched case-control study at the Institute for Child Health Research, West Perth, and the Department of Neonatology, Princess Margaret Hospital for Children, Subiacco, Western Australia. Of 89 cases studied, 42 met criteria for moderate or severe neonatal encephalopathy: severe NE -mechanical ventilation required for $>24$ hours, multiple anticonvulsants, coma, or death; moderate NE: -neurologic abnormalities or seizures requiring anticonvulsants, but resolving before discharge. The estimated incidence of NE in the first week of life was 3.75 per 1000 full term live births, and a case fatality of $8 \%$. Intrapartum hypoxia was the cause of NE in only 5 cases, and antepartum factors were more significant and frequent. Maternal vaginal bleeding in pregnancy, physical trauma during pregnancy, maternal thyroxine treatment, and congenital abnormalities were significantly more frequent in NE patients than in controls. Maternal alcohol consumption, smoking during pregnancy, and gestational diabetes were not related to NE. (Adamson SJ et al. Predictors of neonatal encephalopathy in full term infants. BMI 2 September 1995;311:598602). (Respond: Professor Fiona Stanley, Institute for Child Health Research, PO Box 855, West Perth 6872, Western Australia, Australia).

COMMENT. Antepartum factors and preexisting neurologic abnormalities are important in the cause of neonatal encephalopathy occurring in full term infants. Intrapartum hypoxia is significant in only $6 \%$ of cases.

Problems with definitions and classifications of newborn encephalopathy are reviewed in Progress in Pediatric Neurology II, 1994, pp321-2. The clinical features of hypoxic-ischemic encephalopathy are not specific, and similar symptoms may be caused by metabolic disorders, infection or cerebral malformations. 